\title{
Patrones de tabaquismo en jóvenes y práctica de actividad físico-deportiva durante el tiempo libre Youth smoking patterns and leisure-time physical activity
}

\author{
*Francisco Ruiz Juan, *Ernesto de la Cruz Sánchez, *Jorge Ruiz-Risueño Abad, *María Elena García Montes \\ Universidad de Murcia (España)
}

\begin{abstract}
Resumen: El presente estudio aborda la relación entre dos factores del estilo de vida que tienen una especial incidencia en la salud de los jóvenes, como son la práctica habitual de ejercicio físico y el consumo de tabaco. Para ello, se evaluaron en 6362 estudiantes de Enseñanza Secundaria Obligatoria (ESO) y Post-obligatoria (ESPO) de ambos sexos (3129 varones y 3233 mujeres) el consumo de tabaco y la cantidad habitual de actividad física. Los resultados muestran que los adolescentes estudiados tienen patrones de comportamiento similares al resto de la población española en lo que se refiere al consumo habitual de tabaco y práctica de actividad física cotidiana. En lo que se refiere a la relación entre ambos hábitos, existen diferencias en el consumo habitual de tabaco en función de la cantidad e intensidad de actividad físico-deportiva realizada durante el tiempo libre.
\end{abstract}

Palabras clave: Tabaco; Adolescentes; Deporte; Estilo de vida.

Abstract: This study addresses the relationship between two lifestyle factors that have a particular impact on the health of young people, as are the usual practice of leisure-time physical activity and smoking. Smoking habits and leisure-time physical activity was assessed in a sample of 6362 high school students (3129 men and 3233 women) from Compulsory Secondary Education (ESO) and Post-compulsory Secondary Education (ESPO). The results show that patterns of smoking and leisure-time physical activity of our sample are similar to the rest of the Spanish adolescents. Regarding to the relationship between the two factors studied, differences in smoking habits depending on the quantity and intensity of leisure-time physical activity. Key words: Smoking; Adolescents; Sports; Lifestyle.

\section{Introducción}

Dentro de lo que contemplamos como conductas del estilo de vida nocivas para la salud, encontramos que el tabaquismo es una de las más perjudiciales, siendo la droga legal más consumida en nuestro país, así como en el resto del mundo. El tabaco es causa, aproximadamente, del $16 \%$ de las muertes que suceden en España (Banegas - Banegas, 2001) aunque en los últimos años se viene observando una reducción notable de la mortalidad asociada al tabaquismo en nuestro país que, aunque es positiva, no significa que este hábito haya dejado de ser uno de los principales problemas de salud pública de la sanidad española(Banegas et al., 2005).

Los adolescentes componen el colectivo más vulnerable en lo que a tabaquismo se refiere, ya que es en estas edades cuando se inicia la adicción al consumo de tabaco y son, en conjunto, objetivo de la industria tabacalera para sustituir a los exfumadores, tanto a aquellos que dejan de ser adictos como a los que mueren como consecuencia de este hábito. Además, hay que tener en cuenta que la edad influye en la adicción a esta sustancia: alrededor del $90 \%$ de los fumadores adultos comenzaron a fumar durante la adolescencia, siendo menos probable adquirir este hábito durante la etapa adulta. La población joven española presenta un índice alto de consumo de tabaco: el $46,1 \%$ de los estudiantes afirma haberlo probado alguna vez, un $34 \%$ haberlo fumado en los últimos 12 meses y un $27,8 \%$ en los últimos 30 días. El porcentaje de fumadores diarios es de un $14,8 \%$, porcentaje que asciende al $29,6 \%$ entre los estudiantes de 18 años, siendo ellas las que más consumen $(16,9 \%$ de las mujeres frenteal 12,5\% de los varones; Ministerio de Sanidad y Consumo, 2007).

EnEspaña, la edad de inicio al tabaco suele rondar entre los 11 y los 15 años. De entre las causas que motivan el consumo de tabaco entre los adolescentes, podemos encontrar con más frecuencia las siguientes (Caballero-Hidalgo, González, Pinilla \& Barber, 2005):

- Consumo por parte de los iguales, deseo de encajar o emular al

líder del grupo. El consumo por parte de otros significativos

Fecha recepción: 15-05-08 - Fecha envío revisores: 15-05-08 - Fecha de aceptación: 21-06-08 Correspondencia: Francisco Ruiz Juan

C/Cabo Vidio, 27

30730 San Javier - Murcia (España)

E-mail: fruizj@um.es pertenecientes al entorno próximo (padre, madre, hermanos) no afecta en tanta medida al consumo de cigarrillos.

- Consumo junto con otras sustancias nocivas, dentro de las cuales la que más se asocia al tabaquismo es el alcohol. Es frecuente la simultaneidad en el inicio al consumo en ambas sustancias en zonas de ocio.

- Dinero y disponibilidad de los cigarrillos: el presupuesto de un adolescente puede repercutir en la adquisición o no del hábito; un aumento del coste del tabaco repercute en un descenso notable en el consumo de tabaco entre los niños y adolescentes.

- La educación y el interés por los estudios: a mayor interés por la vida académica y el colegio se observa un descenso del consumo de tabaco entre los jóvenes.

- Otros factores, como la presencia del tabaco en los medios de comunicación, la publicidad o la atracción por el diseño de los envoltorios de cigarrillos por el fabricante, pueden motivar, aunque en menor medida, la adopción de este hábito.

En cuanto a la prevención del tabaquismo en jóvenes y adolescentes, las campañas meramente informativas no suelen dar resultados significativos en este sentido. Funcionan, en mejor medida, los que tienen entre sumetodología programas de educación afectivay estrategias para soportar la presión social, identificación de las consecuencias negativas de este hábito a corto plazo y, sobre todo, la propuesta de alternativas y ocio saludable (Fernández, Nebot \& Jané, 2002). Todas estas características hacen que el profesorado sea un elemento mediador imprescindible en la educación y prevención del tabaquismo en los jóvenes, máxime si se trata desde el área de la Educación Física escolar, ya que bajo el marco descrito anteriormente puede resultar un entorno muy favorable para implementar estrategias de prevención del tabaquismo en niños y adolescentes.

La actividad físico-deportiva durante el tiempo libre podría desempeñar un rol bastante significativo en la prevención del tabaquismo en los adolescentes y jóvenes, ya que la misma parece limitar el consumo de tabaco en las personas que habitualmente la realizan (Hedman, BjergBäcklund, Perzanowski, Sundberg \& Rönmark, 2007). De la misma forma, llevar a cabo actividades físico-deportivas durante el tiempo libre no sólo puede prevenir la adopción de estas conductas nocivas, sino que además puede servir de terapia sustitutiva y paliar la ansiedad que conlleva dejar de fumar y otras consecuencias negativas de este hábito perjudicial, como puede ser la ganancia de peso que se produce en los exfumadores (Stamford, Matter, Fell \& Papenek, 1986). 
El objetivo del presente trabajo es evaluar la conducta respecto al tabaco en adolescentes y su relación con los comportamientos hacia la práctica deportiva y la frecuencia e intensidad de la actividad físicodeportiva realizada durante el tiempo libre.

\section{Método}

La muestra estaba compuesta por 6362 estudiantes de Enseñanza Secundaria Obligatoria (ESO) y Post-obligatoria (ESPO) de ambos sexos (3129 varones y 3233 mujeres) pertenecientes a las provincias de Almería, Granada y Murcia, siendo esta representativa en cada una de ellas (?3\% error; $95.5 \%$ nivel de confianza).

La recogida de los datos se efectuó por medio de dos cuestionarios previamente validados en los que se evaluaba el consumo habitual de tabaco (Pérez-Milena etal., 2007) y la práctica de actividad fisica habitual en lo que se denomina «índice finlandés de actividad física» (Raitakari et al., 1994). En el primero de ellos se les solicitaba a los alumnos que indicaran la frecuencia con la que consumen tabaco, teniendo cinco opciones de respuesta: «habitual», «ocasional», «sólo fines de semana», «lo he dejado» $\mathrm{y}$ «nunca». Posteriormente, los fumadores debían indicar cuántos cigarrillos fuman al día de promedio. Para realizar este capítulo hemos agrupado el consumo en categorías de cinco cigarrillos: «entre 1$5 »$, «entre 6-10», «entre 11-15» y «más de 15».

La cantidad de actividad fisico-deportiva habitual se presenta en tres categorías en función de su intensidad, frecuencia y duración y la relación de la misma con las recomendaciones para mantener la salud en este grupo de edad: insuficiente, ligera y vigorosa o intensa (Raitakari et al., 1994). Para cada uno de estos factores realizamos las comparaciones siguientes según sexo, edad, implicación en la práctica habitual de actividad actividad fisico-deportiva durante el tiempo libre (practicantes, abandono, nunca).

\section{Resultados}

\subsection{Frecuencia y unidades de consumo}

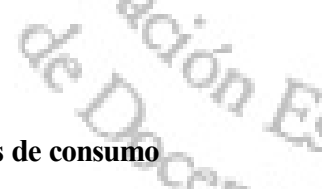

Podemos observar como, en ESO, existe un elevado número de estudiantes que nunca han consumido tabaco, existiendo un descenso de casi 20 unidades porcentuales al pasar a la etapa de ESPO. Este descenso es visible en las tres provincias, al igual que se observa un aumento, de más del doble, en el número de estudiantes que afirman ser fumadores habituales al pasar de una etapa educativa a otra (figura 1). Por otro lado, es importante reseñar que el consumo ocasional y en fines de semana asciende a más del diez por ciento, lo que nos está dando una cifra nada desdeñable de sujetos que se pueden convertir en fumadores habituales en un futuro.

Por consiguiente, existe una relación entre el consumo de tabaco y la edad de los estudiantes de Secundaria, hecho preocupante ya que se observa como el consumo de tabaco se implanta como un hábito en edades muy tempranas de la adolescencia, en la etapa de ESPO existe ya un $30 \%$ de fumadores, situación generalizable a las tres provincias.

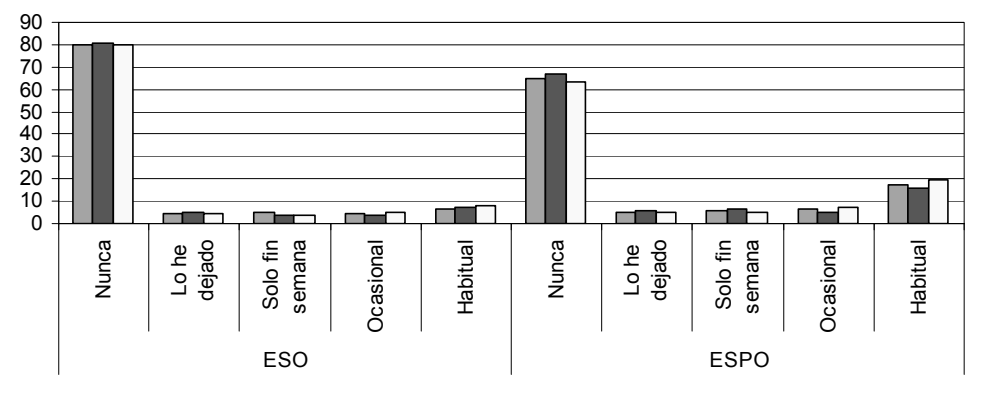

$\square$ Almería $\square$ Granada $\square$ Murcia

Figura 1. Conducta hacia el consumo de tabaco, en las tres provincias y en las dos etapas educativas

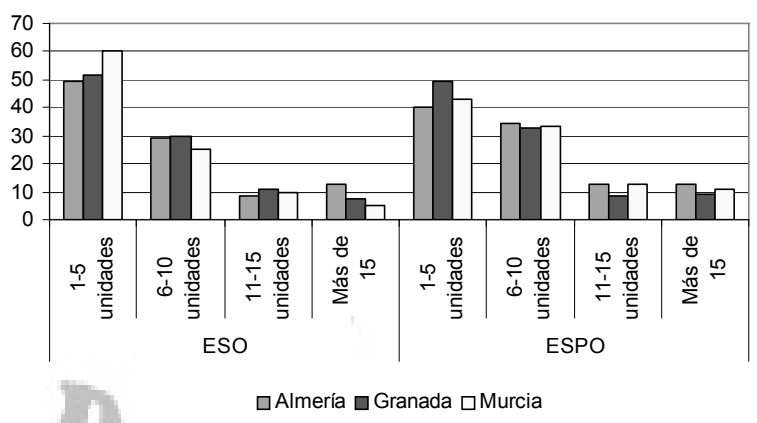

Figura 2. Número de cigarrillos consumidos por los sujetos fumadores, en las tres provincias, en función de la etapa educativa

Por otro lado, encontramos un incremento de cigarrillos consumidos al pasar de ESO a ESPO, debido al descenso en un $10 \%$ del número de sujetos que afirman fumar entre 1-5 cigarrillos diarios. Este descenso se ve plasmado, en ESPO, en el consumo de 6-10 cigarrillos, el cual aumenta en las tres provincias entre un tres y un ocho por ciento. Otro dato bastante preocupante es que más del $20 \%$ de los estudiantes fumadores, tanto de ESO cómo de ESPO, afirman consumir más de 10 cigarrillos diarios, con el consiguiente perjuicio para la salud que esto significa a estas edades (figura 2).

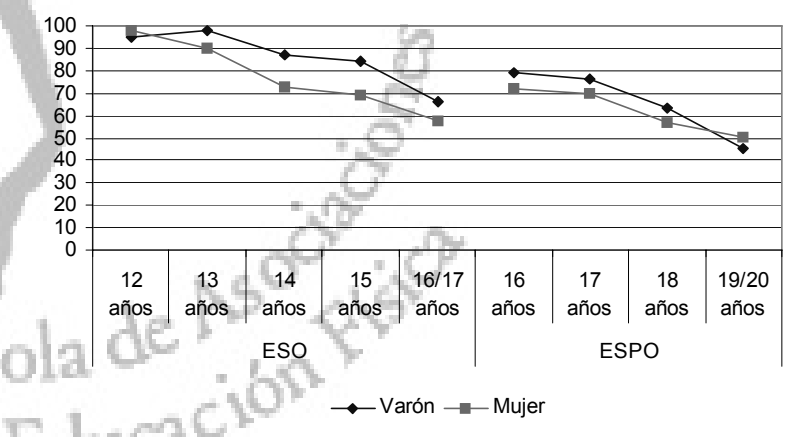

Figura 3. Evolución cronológica del porcentaje de sujetos no fumadores, provincia de Granada

\subsection{Frecuencia y unidades de consumo según sexo y edad}

Es claramente observable una regresión lineal del porcentaje de estudiantes que no consumen tabaco conforme aumenta la edad del sujeto. Podemos ver como, en ESO, casi el 100\% de los sujetos de 12 años afirma no fumar nunca, mientras que en ESPO, a la edad de 19/20 años sólo alrededor del $45 \%$ asegura no hacerlo (figura 3 ).

Sólo encontramos diferencias significativas, en cuanto el sexo, en ESO a partir de los 14 años. En aquellos sujetos que dicen no fumar nunca, so observa como existe mayor porcentaje de varones que se declaran no fumadores que de mujeres. Es ESPO estas diferencias, aunque existen, son más reducidas.

Si observamos la figura 4 podemos ver como ocurre el fenómeno al contrario, se produce una progresión del consumo habitual de tabaco conforme la edad del sujeto va en aumento. Mientras que a la edad de 12 y 13 años casi ningún estudiante de ESO dice fumar tabaco de manera habitual, en ESPO, a la edad de 19/20 años, entre el 25 y el $40 \%$ asegura hacerlo.

Por otro lado, son las mujeres las que en mayor porcentaje fuman cigarrillos habitualmente en ESO con diferencias entre 5 y 15 unidades porcentuales. EnESPO sucede lo mismo, existe un mayor consumo por parte de las mujeres, exceptuando la provincia de Granada que, a los 19/20 años, se produce un cambio brusco, siendo los varones los que consumen más tabaco de manera habitual. 


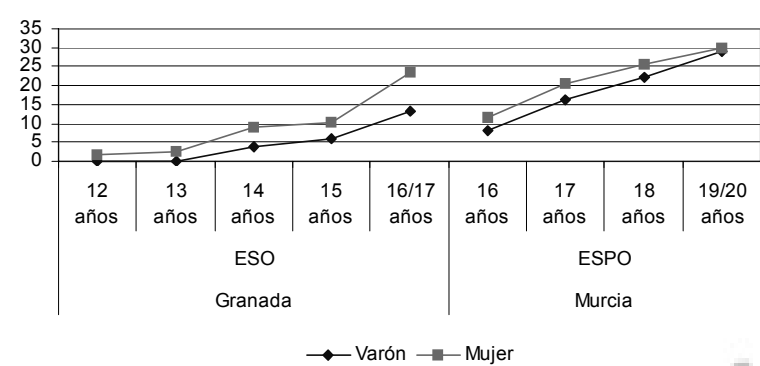

Figura 4. Evolución cronológica del porcentaje de sujetos que son fumadores habituales, provincias de Granada y Murcia

Al analizar las unidades de tabaco consumidas diariamente en relación con la edad y el sexo, nos encontramos con una crecida progresiva del consumo de número de cigarrillos conforme la edad de los estudiantes va en aumento. Este fenómeno sucede en las tres provincias objeto de estudio, siendo más claro en la provincia de Almería dónde observamos que en ESO, a la edad de 12 y 13 años, el $100 \%$ de los sujetos que afirman fumar consumen entre 1 y 5 cigarrillos, mientras que a la edad de 16/17 años se produce un descenso de sesenta unidades porcentuales, para situarse en el $40 \%$, y a los $19 / 20$ años es ya sólo del $20 \%$.

Es a partir de los 13 años cuando se percibe el aumento de cigarrillos consumidos, siendo alrededor del $20 \%$ de los sujetos, de entre 14 y 17 años, los que consumen más de 10 cigarrillos diarios, rondando el $30 \%$ a partir de los 18 años en algunas provincias (figura 5 ).

En cuanto al sexo, no encontramos diferencias que puedan generalizarse a las tres provincias, teniendo los varones y las mujeres casi el mismo consumo hasta los 14 años, pero es cambiante a partir de esta edad sin que las diferencias sean significativas.

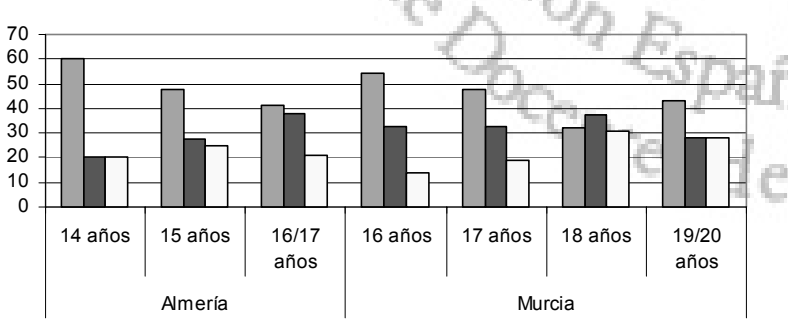

口Entre 1-5 घEntre 6-10 口Más de 10

Figura 5. Porcentaje del número total de cigarrillos consumidos diariamente, en función de la edad, en las provincias de Almería y Murcia

3.3. Frecuencia y unidades de consumo según los comportamientos hacia la práctica físico-deportiva

Podemos apreciar una correlación con diferencias estadísticamente significativas entre la frecuencia de consumo de tabaco y los comportamientos hacia la práctica físico-deportiva, en las tres provincias y en ambas etapas educativas.

Son aquellos sujetos que afirman realizar práctica físico-deportiva en su tiempo libre, los estudiantes que en mayor porcentaje expresan no fumar nunca, estando más de 10 unidades porcentuales por encima de aquellos que aseguran ser inactivos. Por el contrario, se aprecia que los estudiantes sedentarios tienen mayores porcentajes de llevar un consumo habitual de cigarrillos, oscilando la diferencia con los estudiantes activos alrededor del 5\% en ESO y del 10\% en ESPO (figura 6).

Por lo tanto se deduce que existe una correlación positiva, en ambas etapas educativas, entre practicar actividad física y no haber consumido nunca tabaco, de tal forma que son los estudiantes activos los que muestran el mayor porcentaje de no fumadores y los sedentarios los que revelan mayor porcentaje de fumadores habituales.

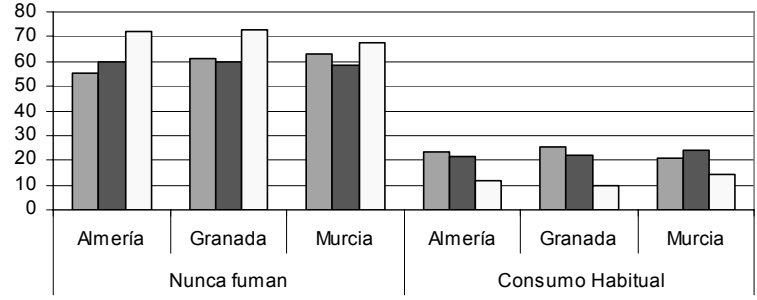

$\square$ Nunca $\square$ Abandono $\square$ Practicantes

Figura 6. Comparación de no fumadores y de fumadores habituales, según los comportamientos ante la práctica físicodeportiva en ESPO

$\mathrm{El}$ análisis de resultados ha manifestado que no se encuentran diferencias estadísticamente significativas ente las unidades de consumo de alcohol y los comportamientos hacia la práctica físico-deportiva, ni en ESO ni en ESPO, en ninguna de las tres provincias objeto de estudio. Por lo tanto, podemos deducir que no existe ninguna relación entre las unidades de cigarrillos consumidos y si realiza práctica físico-deportiva o no.

3.4. Frecuencia y unidades de consumo según el índice finlandés de actividad físico-deportiva

La figura 7 refleja, en las tres provincias y en las dos etapas educativas, como existe mayor porcentaje de no fumadores entre quienes siendo activos realizan la práctica intensa o vigorosa frente a aquellos que la tienen insuficiente+ligera o moderada, diferencias porcentuales que rondan el $10 \%$.

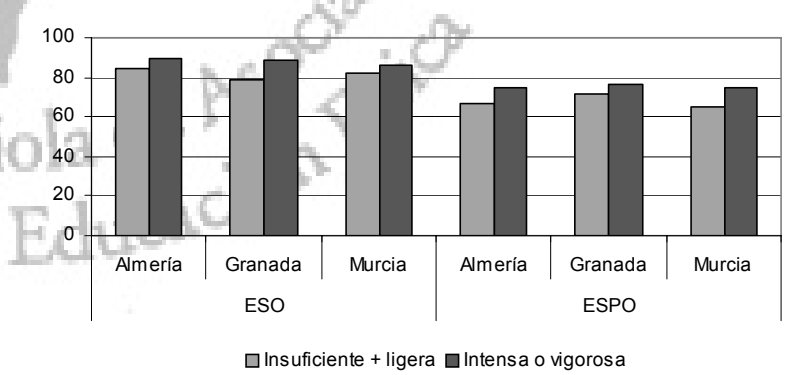

Figura 7. Relación entre el nivel de práctica de actividad físicodeportiva del conjunto de no fumadores, en las tres provincias

Otra relación interesante la podemos encontrar en los fumadores habituales (figura 8) ya que existen diferencias, en algunos casos del más del $10 \%$, entre quienes hacen una práctica insuficiente+ligera y los que la realizan intensa o vigorosa. $\mathrm{O}$ lo que es lo mismo, el porcentaje de fumadores habituales es menor entre quienes tienen un mayor nivel actividad físico-deportiva.

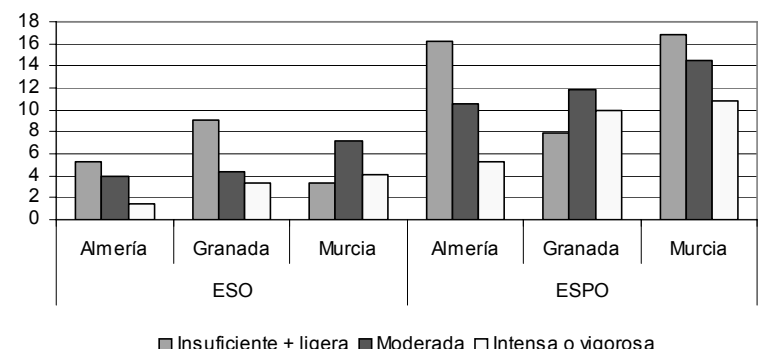

Figura 8. Relación entre el nivel de práctica de actividad físicodeportiva y el consumo habitual de tabaco, en las tres provincias

Sólo se han encontrado diferencias significativas en la provincia de Granada, tanto en ESO como en ESPO (figura 9) en la relación entre las 
unidades de cigarrillos consumidas y el índice de Finlandia. Se observan situaciones diferentes en ambas poblaciones.

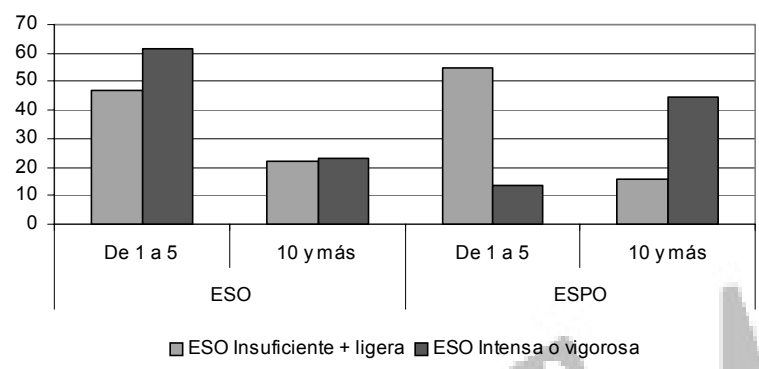

Figura 9. Número de cigarrillos y su relación con el nivel de práctica de actividad físico-deportiva en jóvenes de ESO y ESPO, en la provincia de Granada

\section{Discusión}

Los resultados sugieren que la prevalencia de consumo de alcohol y tabaco en la muestra estudiada es muy similar a la obtenida en la Encuesta Nacional sobre Drogas en España (Ministerio de Sanidad y Consumo, 2007). En el presente trabajo se observa una distribución desigual en el consumo en función del sexo, de la misma forma que lo observado por otros autores en España y en otros países: las mujeres suelen ser fumadoras habituales en mayor medida (Bonet de Luna \& LópezGiménez, 1993; Hedman et al., 2007; Mendoza-Berjano, Batista-Foguet, Sánchez-García \& Carrasco-González, 1998). Parece que, actualmente y desde las dos últimas décadas, se viene observando en muchos países un descenso de la prevalencia del tabaquismo entre los varones y un aumento sustancial de la misma entre el colectivo femenino (Costanza, 2004)

Con el aumento de la edad puede observarse que se incrementa el número de fumadores habituales, así como el número de cigarrillos diarios consumidos, pasando a incrementarse tanto el número de fumadores como la intensidad de la adicción, algo que ha sido observado en otros trabajos (Kelder, Perry, Klepp \& Lytle, 1994)

Existe la creencia generalizada de que la práctica de actividades físico-deportivas supone llevar un estilo de vida más sano (Sygusch, 2005). De hecho, en este estudio podemos observar que, en el caso del tabaquismo, declarar que se realiza práctica de actividad fisica habitual parece estar menos relacionado con el hecho de ser fumador y es que la actitud positiva hacia el ejercicio físico puede ser un elemento que indique una mejor predisposición a llevar un estilo de vida saludable entre los jóvenes.

Muchas de las conductas perjudiciales se asocian unas a otras y esta asociación, entre factores de riesgo para la salud, ha sido observada en numerosos estudios y en ocasiones también incluye la calidad de la dieta, consumo de alcohol, obesidad y otros factores (Burke et al., 1997; Chiolero, Wietlisbach, Ruffieux, Paccaud \& Cornuz, 2006; Galán etal., 2006). Podría decirse que, desde esta perspectiva, la práctica habitual de ejercicio físico en los niveles recomendados para mantener la salud puede ser un indicador más o menos fiable de un estilo de vida saludable, aunque muchos estudios encuentran una asociación positiva entre la práctica deportiva en jóvenes y el consumo de sustancias nocivas y adictivas (Hoffmann, 2006; Moore \& Werch, 2005; Rainey, McKeown, Sargent \& Valois, 1996)

La intensidad de la actividad física habitual supone un elemento diferenciador claro entre los fumadores y los no fumadores, encontrando que la prevalencia de tabaquismo es menos frecuente entre aquellas personas que dicen realizar una práctica de actividad físico-deportiva intensa respecto a los que realizan ejercicio físico de forma moderada o son sedentarios, tal y como señalan otros trabajos al respecto (Nelson \& Gordon-Larsen, 2006). Paradójicamente, entre los individuos que se declaran fumadores parece que encontramos un consumo mayor de cigarrillos entre los sujetos de más edad que realizan práctica de actividad física intensa o vigorosa (figura 15.10), lo que podría apoyar el hecho de algunos estudios que afirman que los jóvenes deportistas mantienen hábitos de consumo de sustancias nocivas en mayor medida incluso que sus pares sedentarios, tal y como hemos señalado anteriormente. No podemos obviar que muchos de los motivos de consumo de sustancias nocivas, como el tabaco o el alcohol, son de índole social y se dan, en mayor medida, entre iguales del entorno próximo, es decir, en sitios donde se establecen relaciones sociales. La «hipótesis del deporte» podría explicar este fenómeno, propuesta por Schuit et al. (2002) que sugieren que la participación en eventos deportivos, sobre todo en los que se practican deportes colectivos, suele conllevar una mayor tasa de consumo de sustancias nocivas, ya que después de participar en los encuentros deportivos suele acudirse a zonas de ocio en las que se fuma y bebe en mayor cantidad (Schuit, van Loon, Tijhuis \& Ocke, 2002). Sería conveniente estudiar las variables y características del deporte practicado por este colectivo con el objetivo de establecer pautas que limiten esta asociación entre deporte y tabaquismo.

Por último, cabe señalar que es necesario interpretar los resultados obtenidos con cierta cautela ya que, aunque el instrumento de medida está validado y contrastado y su uso está extendido en el ámbito de estudios que nos ocupa, hay que señalar que existen trabajos en los que se demuestra que entre el 5,8 y el $8,3 \%$ de los escolares no contestan con sinceridad al evaluar su consumo de tabaco (Barrueco et al., 1999).

\section{Conclusiones}

Con la edad parece que el consumo habitual de tabaco aumenta de forma significativa entre los escolares estudiados. De la misma forma, no sólo aumenta el porcentaje de jóvenes que se declaran fumadores, sino que también existe un consumo más elevado de cigarrillos. En función del sexo, las chicas son en mayor medida fumadoras desde edades más tempranas.

Los comportamientos respecto a la práctica de actividad físicodeportiva pueden predecir en cierta medida el consumo habitual de tabaco, encontrandomenor número de fumadores entre aquellas personas que se declaran practicantes habituales de actividad física.

La intensidad de las actividades realizadas llega a condicionar el consumo habitual de tabaco, existiendo menos fumadores entre el colectivo de jóvenes que afirma realizar actividad física intensa o vigorosa de forma habitual. Sin embargo, entre los individuos fumadores, son los clasificados en ese mismo colectivo (practicantes de actividades físicas vigorosas) los que mayor número de cigarrillos consumen habitualmente.

\section{Referencias}

Banegas Banegas, J. R., Díez Gañán, L., Rodríguez-Artalejo, F., GonzálezEnríquez, J., Graciani Pérez-Regadera,A. \& Villar Álvarez, F. (2001). Mortalidad atribuible al tabaquismo en España en 1998. Medicina Clínica, 117, 692-694.

Banegas, J. R., Díez Gañán, L., González Enríquez, J., VillarAlvarez, F. \& Rodríguez-Artalejo, F. (2005). Recent decrease in smokingattributable mortality in Spain. Medicina Clínica, 28, 769-771.

Barrueco, M., Cordovilla, R., Hernández-Mezquita, M. A., González, J. M., De Castro, J., Rivas, P., et al. (1999). Veracidad en las respuestas de niños, adolescentes y jóvenes a las encuestas sobre el consumo de tabaco realizadas en los centros escolares. Medicine Clinical, 112, 251-254.

Bonet de Luna, C. \& López-Giménez, R. (1993). Alcohol and tobacco consumption of middle school students in the municipality of Madrid. Anales Españoles de Pediatría, 38(1), 49-53.

Burke, V., Milligan, R. A. K., Beilin, L. J., Dunbar, D., Spencer, M., Balde, E., et al. (1997). Clustering of health-related behaviors among 18-year-old australians. Preventive Medicine, 26(5), 724-733.

Caballero-Hidalgo, A., González, B., Pinilla, J. \& Barber, P. (2005). Analysis of factors related to smoking initiation and continued smoking in young adolescents. Gaceta Sanitaria, 19(6), 440-447. 
Chiolero, A., Wietlisbach, V., Ruffieux, C., Paccaud, F. \& Cornuz, J. (2006). Clustering of risk behaviors with cigarette consumption: A population-based survey. Preventive Medicine, 42(5), 348-353.

Costanza, M. (2004). Estimating and approximating prevalence trends. Sozial- und Präventivmedizin, 49, 224-226.

Fernández, S., Nebot, M. \& Jané, M. (2002). Evaluación de la efectividad de los programas escolares de prevención del consumo de tabaco, alcohol y cannabis: ¿qué nos dicen los meta-análisis? [versión electrónica]. Revista Española de Salud Publica, 56(3), 175-187.

Galán, I., Rodríguez-Artalejo, F., Díez-Gañán, L., Tobías, A., Zorrilla, B. \& Gandarillas, A. (2006). Clustering of behavioural risk factors and compliance with clinical preventive recommendations in spain. Preventive Medicine, 42(5), 343-347.

Hedman, L., Bjerg-Bäcklund, A., Perzanowski, M., Sundberg, S. \& Rönmark, E. (2007). Factors related to tobacco use among teenagers. Respiratory Medicine, 101(3), 496-502.

Hoffmann, J. P. (2006). Extracurricular activities, athletic participation, and adolescent alcohol use: gender-differentiated and schoolcontextual effects. Journal of Health and Social Behavior, 47(3), 275-290.

Kelder, S. H., Perry, C. L., Klepp, K. I. \& Lytle, L. L. (1994). Longitudinal tracking of adolescent smoking, physical activity, and food choice behaviors. American Journal of Public Health, 84(7), 1121-1126.

Madrid, Ministerio de Sanidad y Consumo. (2007). Informe de la encuesta estatal sobre uso de drogas en estudiantes de enseñanzas secundarias(ESTUDES) 2006-2007. Madrid: Ministerio de Sanidad y Consumo.

Mendoza-Berjano, R., Batista-Foguet, J. M., Sánchez-García, M. \& Carrasco-González, A. M. (1998). The consumption of tobacco, alcohol and other drugs by adolescent Spanish students. Gaceta Sanitaria, 12(6), 263-271.

Moore, M. J. \& Werch, C. E. (2005). Sport and physical activity participation and substance use among adolescents. Journal of Adolescence Health, 36(6), 486-493.
Nelson, M. C. \& Gordon-Larsen, P. (2006). Physical activity and sedentary behavior patterns are associated with selected adolescent health risk behaviors. Pediatrics, 117(4), 1281-1290.

Pérez-Milena, A., Leal-Helmling, F. J., Jiménez-Pulido, I., MesaGallardo, I., Martínez-Fernández, M. L. \& Pérez-Milena, R. (2007). Evolution of the consumption of toxic substances among the adolescents of an urban area (1997-2004). Atención Primaria, 39(6), 299-304.

Piéron, M., Telama, R., Almond, L. \& Carreiro da Costa, F. (1997). Lifestyle of Young Europeans: Comparative study. In J. Walkuski, S. Wright \& S. Tan Kwang San, Proceedings AIESEP Singapore 1997. World conference on Teaching, Coaching and Fitness Needs in Physical Education and the Sport Sciences (pp. 403-415) Singapore: School of Physical Education, National Institute of Education, Nanyang Technological University.

Rainey, C. J., McKeown, R. E., Sargent, R. G. \& Valois, R. F. (1996). Patterns of tobacco and alcohol use among sedentary, exercising, nonathletic, and athletic youth. Journal of School Health, 66(1), 27-32.

Raitakari, O., Porkka, K., Taimela, S., Telama, R., Rasanen, L. \& Vikari, J. (1994). Effects of persistent physical activity and inactivity on coronary risk factors in children and young adults. American Journal of Epidemiology, 140, 195-205.

Schuit, A. J., van Loon, A. J. M., Tijhuis, M. \& Ocke, M. C. (2002). Clustering of lifestyle risk factors in a general adult population. Preventive Medicine, 35(3), 219-224.

Stamford, B. A., Matter, S., Fell, R. D. \& Papenek, P. (1986). Effects of smoking cessation on weight gain, metabolic rate, caloric consumption and blood lipids. American Journal of Clinical Nutrition, 43, 486-94.

Sygusch, R. (2005). Youth sport-youth health. An overview of the current state of research. Bundesgesundheitsblatt Gesundheitsforschung Gesundheitsschutz, 48(8), 863-872.

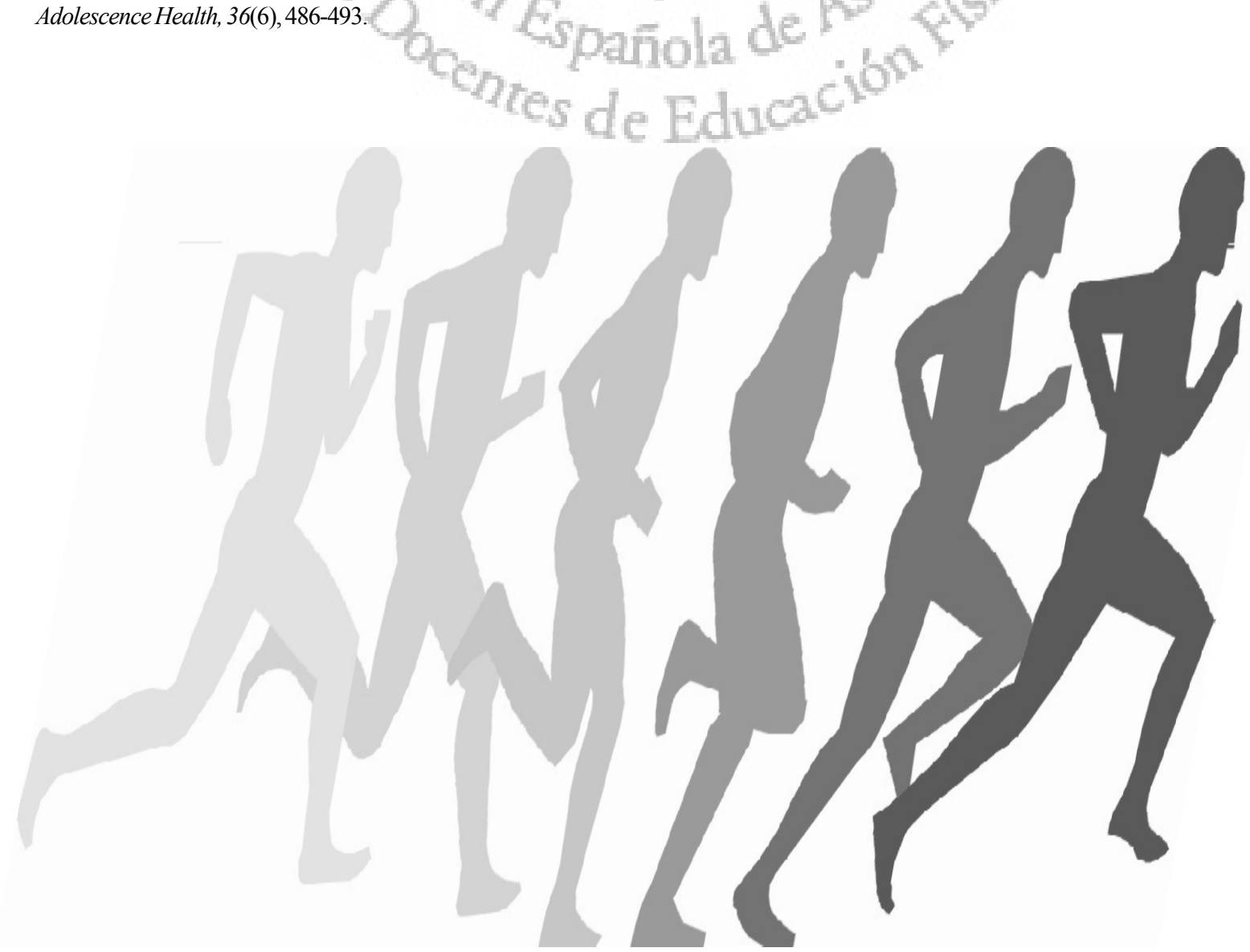

